\title{
All that scatters is not heart: A case of TTP presenting as multi-territorial stroke
}

\author{
Vengadakrishnan Krishnamoorthy' ${ }^{1}$, Shankar Venkatasubramanian², Philo Hazeena ${ }^{2}$, \\ Rithvik Ramesh ${ }^{2}$ \\ ${ }^{1}$ Department of General Medicine, Sri Ramachandra Institute of Higher Education and Research, \\ Chennai, India \\ ${ }^{2}$ Department of Neurology, Sri Ramachandra Institute of Higher Education and Research, Chennai, India
}

\begin{abstract}
Background. Multi-arterial territorial or bi-hemispherical involvement in ischemic stroke, is commonly indicative of an embolic origin from a cardiac source, secondary to either structural or rhythm abnormalities. Other possible sources include acquired and congenietal conditions predisposing to systemic hyper-coagulability. In this regard, thrombotic thrombocytopenic purpura (TTP) a rare multisystem prothrombotic disorder, as a cause of multi-territorial stroke is subsequently discussed.

Case presentation. A 60-year female diabetic on irregular medication presented 30 hrs after sudden diminution of vison in her left eye. MRI brain showed multiple non haemorrhagic infarcts in bilateral parieto-occipital regions and right thalamus with additional multiple scattered infarcts in bilateral cerebellar and frontoparietal regions In view of the multi-territorial and bilateral nature of her stroke, she was considered for therapeutic anticoagulation. But her routine labs done unexpectedly showed haemoglobin of $7.6 \mathrm{~g} / \mathrm{dl}$, platelet count of 35000 platelets per microliter, with elevated creatinine. Subsequent workup revealed evidence of micro-angiopathic haemolysis in her peripheral smear. A provisional diagnosis of acquired TTP was made and the calculated PLASMIC score of the patient was found to be 6 .

Conclusion. We report this case to highlight the need to consider TTP in the differentials when encountering embolic or cryptogenic strokes, and to initiate early plasma exchange.
\end{abstract}

\section{Keywords: thrombotic thrombocytopenic purpura, multi-territorial stroke, cryptogenic stroke}

\section{INTRODUCTION}

Multi-arterial territorial or bi-hemispherical involvement in ischemic stroke, is commonly indicative of an embolic origin from a cardiac source, secondary to either structural or rhythm abnormalities. Other possible sources include acquired and congenietal conditions predisposing to systemic hyper-coagulability. In this regard, thrombotic thrombocytopenic purpura (TTP), a rare multisystem prothrombotic disorder, as a cause of multiterritorial stroke is subsequently discussed. We report a 60-year female diabetic, presenting with progressive cortical blindness, who initially was considered to have a cardioembolic stroke, and was later recognised to have TTP, posing an entirely new set of therapeutic challenges.

\section{CASE REPORT}

A 60 -year female diabetic on irregular medication presented $30 \mathrm{hrs}$ after sudden diminution of vison in her left eye. She had been hospitalised the preceding week with fever and elevated sugars. Presently clinical examination revealed an afebrile female, with Glasgow Coma Scale rate 15\15 and loss of light perception in her left eye. The visual acuity in her right eye could not be conclusively assessed, but she was able to identify objects and colour. MRI brain showed multiple non haemor- 


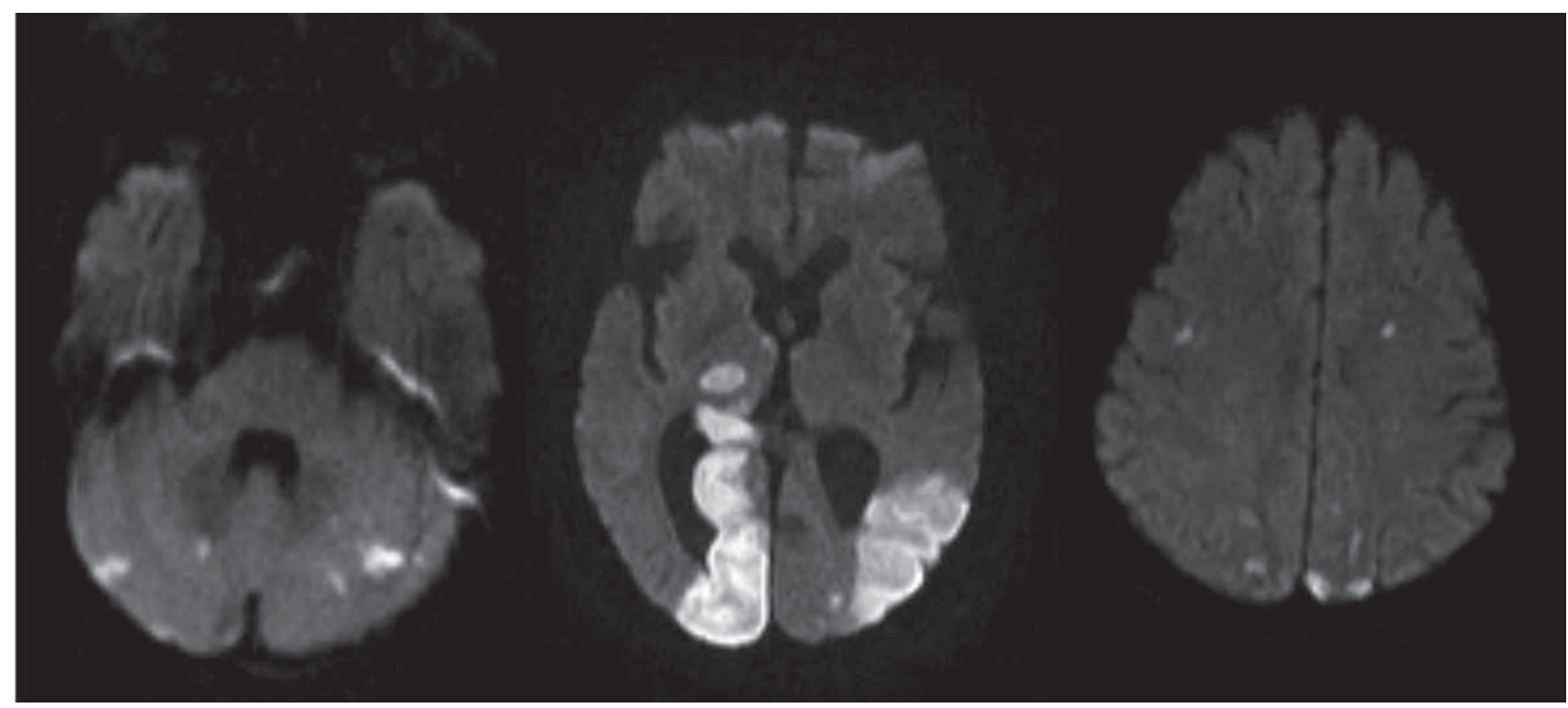

FIGURE 1. MRI brain multiple axial sections showing multiple areas of diffusion restriction in bilateral parietooccipital regions and right thalamus with additional multiple scattered infarcts in bilateral cerebellar and frontoparietal regions

rhagic infarcts in bilateral parieto-occipital regions and right thalamus with additional multiple scattered infarcts in bilateral cerebellar and frontoparietal regions (Figure 1).

In view of the multi-territorial and bilateral nature of her stroke, she was considered for therapeutic anticoagulation considering the possibility of cardio-embolism and was shifted to intensive care. But her routine labs done unexpectedly showed haemoglobin of $7.6 \mathrm{~g} / \mathrm{dl}$, platelet count of 35,000 platelets per microliter, with elevated creatinine, and lactose dehydrogenase levels. Subsequent workup revealed evidence of micro-angiopathic haemolysis in her peripheral smear. A provisional diagnosis of acquired TTP was made and the calculated PLASMIC score of the patient was found to be 6 which was suggestive of severe ADAMST13 dysfunction and its activity $<10$ percent. Measurement of ADAMST13 activity and plasma exchange initiation was not done due to pecuniary causes. Her carotid vertebral doppler, $2 \mathrm{~d}$ echo and 24-hour Holter monitoring were normal. In her further course the patient developed visual loss over her right eye and deep vein thrombosis in her left leg. The patient was referred for further management after high dose steroid initiation.

\section{DISCUSSION}

TTP is an acquired or hereditary prothrombotic syndrome with multisystem abnormalities. It is a female predominant disorder, with incidence upto 4-6 cases per million. Patho-physiologically the underlying defect is in ADAMST13 activity which is a metalloprotease with an important role in cleavage of Von Willebrand factors into functional polymers (1). Clinically, the classical pentad of fever, microangiopathic haemolytic anaemia, thrombocytopenia, renal failure and neurological abnormality develops only rarely and only through the course of the disease (2), but remarkably the present case had all five features.

Neurological involvement in TTP is the cornerstone, with two-thirds presenting with neurological symptoms at onset and nearly all patient developing symptoms during the course of the disease. The spectrum of neurological involvement in TTP includes subtle symptoms such as headache, dizziness, altered sensorium, seizures (26\%), to signs such as aphasia, numbness, weakness, blindness $(41 \%)$ depending on the site involved $(3,4)$.

In particular reference to stroke in TTP, case reports have described ischemic events, ranging from transient ischemic attacks to small and large vessel territories. In TTP, thrombosis, constituted mainly by platelets and von Willebrand factor, usually involves the lacunar arteries leading to multiple cortical and subcortical infarcts mimicking a distal micro-embolic pattern, though large vessel occlusion can also occur (5). TTP also poses diagnostic challenges when the first presentation is stroke without any previous evidence of haemato- 
logical abnormality as observed in the present case. The present case, based on the described imaging features, was being worked up for a cardioembolic source and was being initiated on anticoagulation before lab values arrived. The clinical picture gets more trying when the patient presents with atypical TTP where the blood picture is normal, revealing no clue for the underlying prothrombotic state. Rojas et al. (6), after literature review, report 9 cases including his own, where the onset of stroke preceded the haematological abnormalities. They described that the incipient ADAMTS13 dysfunction may be sufficient to cause local platelet aggregation, without a generalised dysfunction. ADAMTS13 measurement is the definite validation of TTP in both typical and atypical TTP, with levels below 11 being conclusive evidence. But the activity measurement may take days to report and maybe expensive. Plasmic score is a validated surrogate marker of ADAMST13 activity in patients with suspected TTP (7).

Management of stroke in TTP revolves around managing TTP. Plasma exchange is the definitive treatment and should be initiated as early as feasi- ble. Literature review revealed three case reports of patients presenting with TTP associated strokes who were re-perfused. Two cases of atypical TTP improved transiently after thrombolytic therapy (TT) and deteriorated again due to recurrence of thrombosis. One case has haemorrhagic complications too $(8,9)$. The deterioration may be due late initiation of plasma exchange. The third case reported by Boattini et al. (10) was a typical TTP with stroke, and TT was followed promptly by early plasma exchange, which lead to recovery without re-thrombosis. More data is required to establish the role of TT in TTP. Antiplatelets and anticoagulation may be initiated after the platelet count improves beyond 50,000 platelets per microliter (11), though guidelines for definitive management are not clear.

\section{CONCLUSION}

In conclusion, we report this case to highlight the need to consider TTP in the differentials when encountering embolic or cryptogenic strokes, and to initiate early plasma exchange

Conflict of interest: none declared Financial support: none declared

\section{REFERENCES}

1. Terrell DR, Williams LA, Vesely SK, Lammle, B, Hovinga JAK, George JN. The incidence of thrombotic thrombocytopenic purpura-hemolytic uremic syndrome: all patients, idiopathic patients, and patients with severe ADAMTS-13 deficiency. Journal of Thrombosis and Haemostasis. 2005;3(7):1432-1436.

2. Amorosi EL et al. Thrombotic thrombocytopenic purpura - Report of 16 cases and review of the literature. Medicine. 1966:45;139.

3. Meloni, $G$ et al. Thrombotic thrombocytopenic purpura: prospective neurologic, neuroimaging and neurophysiologic evaluation. Haematologica. 2001;86(11):1194-1199.

4. Page EE, Kremer Hovinga JA, Terrell DR, Vesely SK, George JN. Thrombotic thrombocytopenic purpura: diagnostic criteria, clinical features, and long-term outcomes from 1995 through 2015. Blood Adv. 2017 Apr 6;1(10):590-600.

5. Bakshi R, Shaikh ZA, Bates VE, Kinkel PR. Thrombotic thrombocytopenic purpura: brain CT and MRI findings in 12 patients. Neurology. 1999 Apr 12;52(6):1285-8.

6. Rojas JC, Banerjee C, Siddiqui F, Nourbakhsh B, Powell CM. Pearls and oysters: acute ischemic stroke caused by atypical thrombotic thrombocytopenic purpura. Neurology. 2013;80(22):e235-e238.

7. Oliveira DS, Lima TG, Benevides FLN, et al. Plasmic score applicability for the diagnosis of thrombotic microangiopathy associated with ADAMTS13-acquired deficiency in a developing country. Hematol Transfus Cell Ther. 2019;41(2):119-124.

8. Sevy A, Doche E, Squarcioni C, et al. Stroke in a young patient treated by alteplase heralding an acquired thrombotic thrombocytopenic purpura. J Clin Apher. 2011;26:152-5

9. Sevy A, Doche E, Squarcioni C, Poullin P, Serratrice J, Nicoli F, Weiller PJ. Stroke in a young patient treated by alteplase heralding an acquired thrombotic thrombocytopenic purpura. $J$ Clin Apher. 2011;26(3):152-5.

10. Boattini M, Procaccianti G. Stroke due to typical thrombotic thrombocytopenic purpura treated successfully with intravenous thrombolysis and therapeutic plasma exchange. BMJ Case Rep. 2013;2013:bcr2012008426.

11. Matzdorff A, Beer JH. Immune thrombocytopenia patients requiring anticoagulation - maneuvering between Scylla and Charybdis. Semin Hematol. 2013;50(suppl 1):S83-S88. 